\title{
Mogamulizumab treatment of refractory peripheral T-cell lymphoma following autologous stem cell transplantation: A case report
}

\author{
YUKIE FUJITA, AYA NAKAYA, SHINYA FUJITA, MASAAKI HOTTA, HIDEAKI YOSHIMURA, YOSHIKO AZUMA, \\ TAKAHISA NAKANISHI, ATSUSHI SATAKE, KAZUYOSHI ISHII, TOMOKI ITO and SHOSAKU NOMURA
}

First Department of Internal Medicine, Kansai Medical University, Hirakata, Osaka 573-1010, Japan

Received May 20,2015; Accepted November 23, 2015

DOI: $10.3892 / \mathrm{mco} .2015 .695$

\begin{abstract}
Peripheral T-cell lymphoma (PTCL) represents a small heterogeneous group of non-Hodgkin lymphoma (NHL) that accounts for $\sim 10 \%$ of NHLs in western countries and $25 \%$ of NHLs in Japan. The disease remains extremely difficult to treat. Therefore, novel treatment modalities are required. Mogamulizumab is a humanized immunoglobulin G1 monoclonal antibody that targets $\mathrm{CC}$ chemokine receptor 4 . To the best of our knowledge, the efficacy of mogamulizumab in patients who are refractory to conventional chemotherapy following autologous stem cell transplantation has not been investigated previously. The present study reports a patient with PTCL who relapsed following autologous stem cell transplantation and became resistant to salvage chemotherapy, in whom mogamulizumab showed evident efficacy without severe adverse event.
\end{abstract}

\section{Introduction}

Peripheral T-cell lymphoma (PTCL) represents a small heterogeneous group of non-Hodgkin lymphoma (NHL) that accounts for $\sim 10 \%$ of NHLs in western countries and $\sim 25 \%$ of NHLs in Japan $(1,2)$. PTCLs generally have a poor outcome with shorter long-term survival compared with B-cell lymphomas. For example, the 5-year overall survival for the World Health Organization (WHO) subtype 'PTCL-not otherwise specified' (PTCL-NOS) is $32 \%$. PTCL remains extremely difficult to treat as the majority of subtypes become refractory to even aggressive chemotherapy regimens or relapse, and thus, there is a requirement for novel treatment modalities. Mogamulizumab is a humanized immunoglobulin G1 monoclonal antibody that targets $\mathrm{CC}$ chemokine receptor 4 (CCR4).

Correspondence to: Dr Aya Nakaya, First Department of Internal Medicine, Kansai Medical University, 2-5-1 Shin-machi, Hirakata, Osaka 573-1010, Japan

E-mail: nakaya1016@yahoo.co.jp

Key words: mogalimuzumab, chemokine receptor 4 antibody, peripheral T-cell lymphoma, autologous stem cell transplantation
CCR4 is highly expressed by aggressive PTCLs, particularly adult T-cell leukemia/lymphoma (ATL) and cutaneous T-cell lymphomas (CTCLs). A phase II study of mogamulizumab yielded an objective response in $35 \%$ of patients and a complete response in $14 \%$, with a median progression-free survival (PFS) of 3 months (3). To date, however, the efficacy of mogamulizumab in patients who become refractory to chemotherapy following autologous stem cell transplantation (ASCT) has not been investigated.

The present study reports a patient with PTCL who became refractory following ASCT and resistant to a salvage therapy, in whom mogamulizumab showed evident efficacy without severe adverse event (AE).

\section{Case report}

A 49-year-old woman presented with subcutaneous tumors and erythema from face to trunk with cervical lymphadenopathy. From a biopsy of the tumor, the patient was diagnosed with PTCL-NOS, clinical stage IIIA. The patient received 5 cycles of a regimen containing cyclophosphamide, doxorubicin, vincristine and prednisone, and achieved complete remission (CR). The patient subsequently received an ASCT using the ranimustine/etoposide/cytarabine/melphalan regimen. Following ASCT, the patient remained in CR for 1 month before the skin lesions worsened without lymphadenopathy. The patient received the rituximab, etoposide, methylprednisolone, cytarabine and cisplatin regimen, as a salvage therapy, but the skin lesions remained refractory. The patient received phototherapy, which was similarly ineffective. Re-biopsy of the skin revealed that it was positive for CCR4 (Fig. 1). The patient received mogamulizumab once a week for 8 weeks by intravenous infusion at $1.0 \mathrm{mg} / \mathrm{kg}$. A grade 1 infusion reaction was observed at the first dose; however, no other AE was observed. After 5 weeks, the skin lesions had improved, and after 8 weeks the patient achieved CR (Fig. 2). The patient remained in CR for $>1$ year.

\section{Discussion}

According to the WHO classification, PTCL is a heterogeneous category of mature T-cell neoplasms. The most common 
A

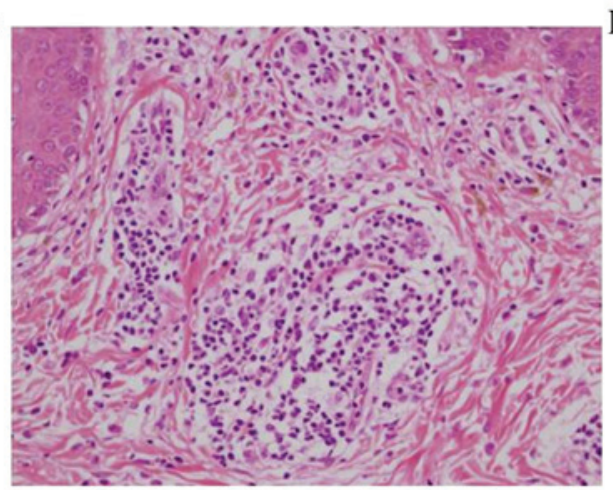

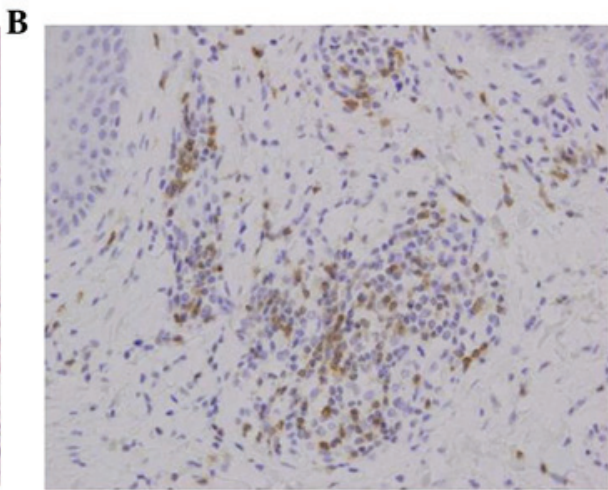

Figure 1. (A) Hematoxylin and eosin stain of a skin biopsy specimen showing dense infiltration of atypical lymphocytes (original magnification, $\mathrm{x} 200$ ). (B) Immunohistochemically, these lymphocytes were positive for CC chemokine receptor 4 (original magnification, x200).
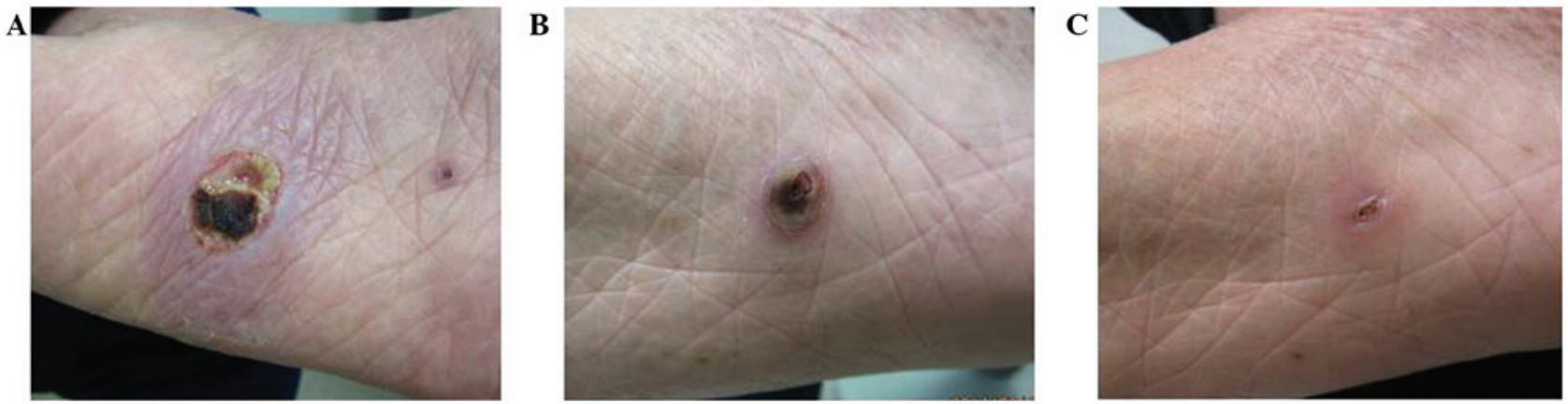

Figure 2. (A) Prior to treatment with mogamulizumab there was a cutaneous nodule on the patient's sole. (B) The cutaneous lesion appeared to improve after 5 cycles of mogamulizumab. (C) The cutaneous lesion almost disappeared after 8 cycles of mogamulizumab.

mature T-cell neoplasms are PTCL-NOS, angioimmunoblastic T-cell lymphoma anaplastic large-cell lymphoma (ALCL) (4). PTCL remains extremely difficult to treat, as the majority of PTCL subtypes become refractory to even aggressive chemotherapy regimens or relapse, and thus there is a medical requirement for novel treatment modalities.

Mogamulizumab is the first approved glycol-engineered therapeutic antibody and first approved monoclonal antibody to target CCR4 (5-7). CCR4 is principally expressed on regulatory $\mathrm{T}$ cells (Tregs) and helper $\mathrm{T}$ cells where it functions to induce homing of these leukocytes to sites of inflammation. Tregs have an essential role in providing and maintaining a favorable environment in which tumors can grow. Mogamulizumab depletes CCR4-positive Tregs, potentially evoking antitumor immune responses by autologous effector cells. This ability is highly pertinent as subsets of malignant $\mathrm{T}$ cells are believed to function as CD4-positive Tregs, overexpressing CCR4 (8-10). CCR4 is highly expressed by PTCLs, particularly ATL and CTCLs. A previous study of 169 biopsies demonstrated that CCR4 was expressed in the following PTCL subtypes: ALCL anaplastic large cell kinase (ALK)-negative (67\%), PTCL-NOS (38\%), angioimmunoblastic T-cell lymphoma (35\%), ALCL ALK-positive (4.2\%), nasal-type natural killer/T-cell lymphoma (3.7\%). Within the PTCL-NOS subtype, CCR4-positive patients had a significantly poorer prognosis compared to CCR4-negative patients and multivariate analysis confirmed that CCR4 expression was an independent unfavorable prognostic factor (11).
Mogamulizumab is developed, owned and manufactured by Kyowa Hakko Kirin Co., Ltd. (Tokyo, Japan). The drug was approved in Japan for the treatment of patients with relapsed/refractory ATL in March 2012 and for the treatment of relapsed/refractory CCR4-positive PTCL and CTCL in March 2014. This agent is currently only available in Japan.

In a phase I study conducted in patients with relapsed ATL and PTCL/CTCL, mogamulizumab was well tolerated at doses of $\leq 1.0 \mathrm{mg} / \mathrm{kg}$. The overall response rate (ORR) in the 16 patients included in this study was $31 \%$ (12). Similarly, a recent phase I/II multicenter, dose-escalation study of mogamulizumab in relapsed patients with CTCL that was conducted in the United States reported an ORR of 36.8\% (13). A Japanese multicenter phase II study investigating mogamulizumab in patients previously treated with PTCL or CTCL was published in 2014. In the 37 patients who received mogamulizumab, the ORR was $35 \%$, complete response was $14 \%$ and median PFS was 3 months (3). Based on the results of this phase II study, mogamulizumab was approved for use of refractory/relapsed PTCL in Japan.

In the Japanese phase II study, AEs were mild and reversible. The most common of these were hematological events (lymphocytopenia, 81\%; leukocytopenia, 43\%; thrombocytopenia, $8 \%$; neutropenia, 38\%), followed by skin and subcutaneous tissue disorders (51\%) (3). The patient in the present study had an infusion reaction, which was observed in $24 \%$ of patients in the phase II study. Due to the low frequency of this disease and its endemic features, few physicians have 
used this agent. Studies that became the foundation of its approval were conducted on relatively small cohorts; therefore, there is a requirement for larger multicenter investigations on the use of mogamulizumab to treat PTCL. The present case is of particular interest, as to the best of our knowledge, the use of mogamulizumab has not been described previously in detail in PTCL cases refractory following ASCT. Three patients who were refractory following ASCT were included in the Japanese phase II study; however, full details of these cases were not included (3). The present case became resistant quickly following ASCT and remained refractory to a salvage therapy, but mogamulizumab monotherapy was markedly effective. In our experience, mogamulizumab has promising efficacy with relapsed PTCL patients following ASCT.

Mogamulizumab is likely to provide new and promising treatment options for patients with CCR4-positive T-cell lymphomas; however, the precise approach may require refinement. For example, patients may benefit from the use of mogamulizumab in combination with other agents. Further studies are required to fully understand the physiological effects of this agent and to determine which subtypes of T-cell lymphoma may receive optimum benefit from this agent.

\section{References}

1. No authors listed: The World Health Organization classification of malignant lymphomas in Japan: Incidence of recently recognized entities. Lymphoma Study Group of Japanese Pathologists. Pathol Int 50: 696-702, 2000.

2. Aoki R, Karube K, Sugita Y, Nomura Y, Shimizu K, Kimura Y, Hashikawa K, Suefuji N and Kikuchi M: Distribution of malignant lymphoma in Japan: Analysis of 2260 cases, 2001-2006. Pathol Int 58: 174-182, 2008.

3. Ogura M, Ishida T, Hatake K, Taniwaki M, Ando K, Tobinai K, Fujimoto K, Yamamoto K, Miyamoto T and Uike N: Multicenter phase II study of mogamulizumab (KW-0761), a defucosylated anti-cc chemokine receptor 4 antibody, in patients with relapsed peripheral T-cell lymphoma and cutaneous T-cell lymphoma. J Clin Oncol 32: 1157-1163, 2014.
4. Swerdlow SH, Campo E, Harris NL, et al (eds): WHO Classification of Tumours of Haematolopoietic and Lymphoid Tissues. 4th edition. IARC. Lyon, 2008.

5. Imai T, Nagira M, Takagi S, Kakizaki M, Nishimura M, Wang J, Gray PW, Matsushima K and Yoshie O: Selective recruitment of CCR4-bearing Th2 cells toward antigen-presenting cells by the $\mathrm{CC}$ chemokines thymus and activation-regulated chemokine and macrophage-derived chemokine. Int Immunol 11: 81-88, 1999.

6. Niwa R, Shoji-Hosaka E, Sakurada M, Shinkawa T, Uchida K, Nakamura K, Matsushima K and Ueda R: Defucosylated chimeric anti-CC chemokine receptor $4 \mathrm{IgG} 1$ with enhanced antibody-dependent cellular cytotoxicity shows potent therapeutic activity to T-cell leukemia and lymphoma. Cancer Res 64: 2127-2133, 2004.

7. Ishii T, Ishida T, Utsunomiya A, Inagaki A, Yano H, Komatsu H, Iida S, Imada K, Uchiyama T, Akinaga S, et al: Defucosylated humanized anti-CCR4 monoclonal antibody KW-0761 as a novel immunotherapeutic agent for adult T-cell leukemia/lymphoma. Clin Cancer Res 16: 1520-1531, 2010.

8. Ohshima K, Karube K, Kawano R, Tsuchiya T, Suefuji H, Yamaguchi T, Suzumiya J and Kikuchii M: Classification of distinct subtypes of peripheral T-cell lymphoma unspecified, identified by chemokine and chemokine receptor expression: Analysis of prognosis. Int J Oncol 25: 605-613, 2004.

9. Nakagawa M, Nakagawa-Oshiro A, Karnan S, Tagawa H, Utsunomiya A, Nakamura S, Takeuchi I, Ohshima K and Seto M: Array comparative genomic hybridization analysis of PTCL-U reveals a distinct subgroup with genetic alterations similar to lymphoma-type adult T-cell leukemia/lymphoma. Clin Cancer Res 15: 30-38, 2009.

10. Ishida $\mathrm{T}$ and Ueda R: Immunopathogenesis of lymphoma: Focus on CCR4. Cancer Sci 102: 44-50, 2011.

11. Ishida T, Inagaki H, Utsunomiya A, Takatsuka Y, Komatsu H, Iida S, Takeuchi G, Eimoto T, Nakamura S and Ueda R: CXC chemokine receptor 3 and $\mathrm{CC}$ chemokine receptor 4 expression in T-cell and NK-cell lymphomas with special reference to clinicopathological significance for peripheral T-cell lymphoma, unspecified. Clin Cancer Res 10: 5494-5500, 2004.

12. Yamamoto K, Utsunomiya A, Tobinai K, Tsukasaki K, Uike N, Uozumi K, Yamaguchi K, Yamada Y, Hanada S, Tamura K, et al: Phase I study of KW-0761, a defucosylated humanized anti-CCR4 antibody, in relapsed patients with adult T-cell leukemia-lymphoma and peripheral T-cell lymphoma. J Clin Oncol 28: 1591-1598, 2010.

13. Duvic M, Pinter-Brown L, Foss FM, Sokol L, Jorgensen JL, et al: Phase $1 / 2$ study of mogamulizumab, a defucosylated anti-CCR4 antibody, in previously treated patients with cutaneous T-cell lymphoma. Blood 125: 1883-1889, 2015. 